\title{
THE BEREZIN TRANSFORM AND OPERATORS ON SPACES OF ANALYTIC FUNCTIONS
}

\author{
KAREL STROETHOFF \\ Department of Mathematical Sciences, University of Montana \\ Missoula, Montana 59812-1032, U.S.A. \\ E-mail: ma_kms@selway.umt.edu
}

\begin{abstract}
In this article we will illustrate how the Berezin transform (or symbol) can be used to study classes of operators on certain spaces of analytic functions, such as the Hardy space, the Bergman space and the Fock space. The article is organized according to the following outline.

1. Spaces of analytic functions

2. Definition and properties Berezin transform

3. Berezin transform and non-compact operators

4. Commutativity of Toeplitz operators

5. Berezin transform and Hankel or Toeplitz operators

6. Sarason's example
\end{abstract}

1. Spaces of analytic functions. In this section we will introduce the spaces of analytic functions on which we will be working. We start with the following general definition.

Definition 1.1. A reproducing functional Hilbert space on an open subset $\Omega$ of $\mathbb{C}$ is a Hilbert space $\mathcal{H}$ of functions on $\Omega$ such that for every $w \in \Omega$ the linear functional $f \mapsto f(w)$ is bounded on $\mathcal{H}$.

If $\mathcal{H}$ is a reproducing functional Hilbert space on set $\Omega$, then by the Riesz Representation Theorem for every $w \in \Omega$ there is a unique element $K_{w} \in \mathcal{H}$ for which $f(w)=\left\langle f, K_{w}\right\rangle$, for all $f \in \mathcal{H}$. We call the function $K_{w}$ the reproducing kernel at $w$.

Before we turn to a few examples we will prove some simple results about these reproducing kernels. The following proposition gives a way to compute the reproducing kernels.

1991 Mathematics Subject Classification: Primary 47B07, 47B35; Secondary 30C40, 31A05.

Research of the author supported by summer grants from the University of Montana and the Montana University system.

The paper is in final form and no version of it will be published elsewhere. 
Proposition 1.2. If $\left\{e_{j}: j \in J\right\}$ is an orthonormal basis for the reproducing functional Hilbert space $\mathcal{H}$ of functions on an open set $\Omega \subset \mathbb{C}$, then

$$
K_{w}=\sum_{j \in J} \overline{e_{j}(w)} e_{j}
$$

where the convergence is in $\mathcal{H}$. In particular,

$$
K_{w}(z)=\sum_{j \in J} \overline{e_{j}(w)} e_{j}(z), \quad z \in \Omega
$$

Pr oof. By Parseval's formula,

$$
K_{w}=\sum_{j \in J}\left\langle K_{w}, e_{j}\right\rangle e_{j}=\sum_{j \in J} \overline{\left\langle e_{j}, K_{w}\right\rangle} e_{j}=\sum_{j \in J} \overline{e_{j}(w)} e_{j},
$$

where the convergence is in $\mathcal{H}$. Now, if $z \in \Omega$, the continuity of the linear functional $f \mapsto f(z)$ and the convergence of the above series implies that

$$
K_{w}(z)=\sum_{j \in J} \overline{e_{j}(w)} e_{j}(z)
$$

completing the proof of this proposition.

It follows from the above proposition that $K_{z}(w)=\overline{K_{w}(z)}$. Writing $K(z, w)=K_{w}(z)$, we have $K(w, z)=\overline{K(z, w)}$, for all $z, w \in \Omega$. The norm of $K_{w}$ is easily determined:

$$
\left\|K_{w}\right\|^{2}=\left\langle K_{w}, K_{w}\right\rangle=K_{w}(w)=K(w, w) .
$$

The function

$$
k_{w}=\frac{K_{w}}{\left\|K_{w}\right\|}=\frac{K_{w}}{K(w, w)^{1 / 2}}
$$

is called the normalized reproducing kernel at $w$. We will only consider reproducing functional Hilbert spaces of analytic functions. Note that then for each fixed $w \in \Omega$ the function $z \mapsto K(z, w)$ (that is, the function $K_{w}$ ) is analytic on $\Omega$, while the function $z \mapsto K(w, z)$ (that is, the function $\bar{K}_{w}$ ) is conjugate-analytic on $\Omega$.

ExAmPLE 1.4. Let $H^{2}$ denote the space of all analytic functions $f$ on the unit disk $\mathbb{D}=\{z \in \mathbb{C}:|z|<1\}$ whose Taylor coefficients are square-integrable. This space is called the Hardy space. Every function in $H^{2}$ has radial (in fact, non-tangential) limits at almost every point of $\mathbb{T}=\{\zeta:|\zeta|=1\}$, that is, for every $f \in H^{2}$ the limit function $f^{*}(\zeta)=\lim _{r \rightarrow 1^{-}} f(r \zeta)$ exists for a.e. $\zeta \in \mathbb{T}$. Furthermore, if $f \in H^{2}$, then $f^{*} \in L^{2}(\mathbb{T})$. Identifying $H^{2}$ with $\left\{f^{*}: f \in H^{2}\right\}$, we regard the space $H^{2}$ as a linear subspace of $L^{2}(\mathbb{T})$ with inner product given by

$$
\langle f, g\rangle=\int_{0}^{2 \pi} f\left(e^{i \theta}\right) \overline{g\left(e^{i \theta}\right)} \frac{d \theta}{2 \pi}, \quad f, g \in L^{2}(\mathbb{T}) .
$$

It follows from the inequality

$$
|f(w)| \leq\|f\|\left(1-|w|^{2}\right)^{-1 / 2}
$$

valid for $f \in H^{2}$ and $w \in \mathbb{D}$, that $H^{2}$ is a reproducing functional Hilbert space on $\mathbb{D}$. The set $\left\{z^{n}: n \geq 0\right\}$ is an orthonormal basis for $H^{2}$, and thus

$$
K_{w}(z)=\sum_{n=0}^{\infty} \overline{w^{n}} z^{n}=\frac{1}{1-\bar{w} z}, \quad z, w \in \mathbb{D} .
$$


Example 1.6. The Bergman space $L_{a}^{2}$ is the space of analytic functions on $\mathbb{D}$ which are square-integrable with respect to Lebesgue measure on $\mathbb{D}$. Writing $A$ to denote the Lebesgue measure on $\mathbb{D}$, normalized so that $\mathbb{D}$ has measure $1, L_{a}^{2}$ is a linear subspace of $L^{2}(\mathbb{D})$ with inner product given by

$$
\langle f, g\rangle=\int_{\mathbb{D}} f(z) \overline{g(z)} d A(z), \quad f, g \in L^{2}(\mathbb{D}) .
$$

If the analytic function $f$ on $\mathbb{D}$ has power series expansion $f(z)=\sum_{n=0}^{\infty} a_{n} z^{n}$, then

$$
\|f\|^{2}=\int_{\mathbb{D}}|f(z)|^{2} d A(z)=\sum_{n=0}^{\infty} \frac{\left|a_{n}\right|^{2}}{n+1} .
$$

For fixed $w \in \mathbb{D}$, using Cauchy-Schwarz we have

$$
\sum_{n=0}^{\infty}\left|a_{n}\right||w|^{n} \leq\left(\sum_{n=0}^{\infty}(n+1)|w|^{2}\right)^{1 / 2}\left(\sum_{n=0}^{\infty} \frac{\left|a_{n}\right|^{2}}{n+1}\right)^{1 / 2}
$$

and thus

$$
|f(w)| \leq\|f\|\left(1-|w|^{2}\right)^{-1}
$$

It follows from the above inequality that $L_{a}^{2}$ is a closed linear subspace of $L^{2}(\mathbb{D})$, and thus $L_{a}^{2}$ is a reproducing functional Hilbert space on $\mathbb{D}$. The set $\left\{(n+1)^{1 / 2} z^{n}: n \geq 0\right\}$ is an orthonormal basis for $L_{a}^{2}$, and thus

$$
K_{w}(z)=\sum_{n=0}^{\infty} \overline{e_{n}(w)} e_{n}(z)=\sum_{n=0}^{\infty}(n+1) \bar{w}^{n} z^{n}=\sum_{n=0}^{\infty}(n+1)(\bar{w} z)^{n} .
$$

Differentiating $\sum_{n=0}^{\infty} x^{n}=(1-x)^{-1}$ we see that $\sum_{n=0}^{\infty}(n+1) x^{n}=(1-x)^{-2}$ and thus obtain the following formula for the Bergman reproducing kernel:

$$
K_{w}(z)=\frac{1}{(1-\bar{w} z)^{2}}, \quad \text { for } w, z \in \mathbb{D} .
$$

ExAmple 1.9. The Fock space (or Segal-Bargmann space) is the space of entire functions that are square-integrable with respect to Gaussian measure on the complex plane, that is, the space of all analytic functions $f$ on $\mathbb{C}$ for which $\int_{\mathbb{C}}|f(z)|^{2} d \mu(z)<\infty$, where $d \mu(z)=e^{-|z|^{2} / 2} d A(z) / 2$. The space $\mathcal{F}$ is a closed linear subspace of the space $L^{2}(\mathbb{C}, d \mu)$ with inner product given by

$$
\langle f, g\rangle=\int_{\mathbb{C}} f(z) \overline{g(z)} d \mu(z), \quad f, g \in L^{2}(\mathbb{C}, d \mu),
$$

and thus is a Hilbert space. The functions $z^{n}(n \geq 0)$ are orthogonal in $\mathcal{F}$ and their linear span is clearly dense in $\mathcal{F}$. Using polar coordinates we see

$$
\begin{aligned}
\left\|z^{n}\right\|_{\mathcal{F}}^{2} & =\int_{\mathbb{C}}|z|^{2 n} e^{-|z|^{2} / 2} d A(z) / 2=\int_{0}^{\infty} \int_{0}^{2 \pi} r^{2 n+1} e^{-r^{2} / 2} d r d \theta / 2 \pi \\
& =\int_{0}^{\infty} x^{n} e^{-x / 2} d x / 2=2^{n} \int_{0}^{\infty} t^{n} e^{-t} d t=n ! 2^{n} .
\end{aligned}
$$


Thus $\left\{z^{n} /\left(n ! 2^{n}\right)^{1 / 2}: n \geq 0\right\}$ is an orthonormal basis in $\mathcal{F}$, and hence the reproducing kernel in $\mathcal{F}$ is given by

$$
K_{w}(z)=\sum_{n=0}^{\infty} \overline{e_{n}(w)} e_{n}(z)=\sum_{n=0}^{\infty} \frac{(\bar{w} z / 2)^{n}}{n !}=e^{\bar{w} z / 2} .
$$

2. Definition and properties of the Berezin transform. In this section we define the Berezin transform, discuss some elementary properties of the Berezin transform, and give some preliminaries for the spaces of analytic functions introduced in the previous section.

Definition 2.1. Let $\mathcal{H}$ be a reproducing functional Hibert space on an open subset $\Omega$ of $\mathbb{C}$. If $S$ is a bounded linear operator on $\mathcal{H}$, the Berezin transform of $S$ is defined by

$$
\widetilde{S}(w)=\left\langle S k_{w}, k_{w}\right\rangle, \quad \text { for } w \in \Omega .
$$

Note that the function $\widetilde{S}$ is bounded on $\Omega$.

TheOREm 2.2. Let $\mathcal{H}$ be a reproducing functional Hibert space of analytic functions on an open subset $\Omega$ of $\mathbb{C}$ and let $S$ be a bounded operator on $\mathcal{H}$. Then

$$
S=0 \Leftrightarrow \widetilde{S}(w)=0, \quad \text { for all } w \in \Omega .
$$

Before we will prove the above theorem we will need a result on the zero set of an analytic function of more than one variable. Recall that the zeros of an analytic function of one complex variable are isolated. This is no longer true when we consider analytic functions of several complex variables, for example, the zeros of the function $h\left(z_{1}, z_{2}\right)=z_{1}$ form the subset $\left\{\left(0, z_{2}\right): z_{2} \in \mathbb{C}\right\}$ of $\mathbb{C}^{2}$. Thus, varieties of analytic functions can be quite large (for example uncountable). The following lemma does provide a restriction of the zeros of an analytic function of more than one variable, and will be a useful tool in proving Theorem 2.2. In this lemma and the proof of Theorem 2.2 we will use the following notation: given $\Omega \subset \mathbb{C}$ we write $\Omega^{*}=\{\bar{w}: w \in \Omega\}$. Note that $\Omega^{*}$ is open if $\Omega$ is an open subset of $\mathbb{C}$.

LEMma 2.3. Let $\Omega$ be an open subset of $\mathbb{C}$. If $h$ is analytic on $\Omega \times \Omega^{*}$ and $h(z, \bar{z})=0$, for all $z \in \Omega$, then $h=0$.

Proof. Without loss of generality we may assume that $0 \in \Omega$. Let $\varrho>0$ be such that $D=\{z \in \mathbb{C}:|z|<\varrho\} \subset \Omega$. Then $h=\sum_{m=0}^{\infty} h_{m}$ on $D \times D$, where each $h_{m}$ is a homogeneous polynomial of degree $m$ on $\mathbb{C} \times \mathbb{C}$. For $-1<t<1, z \in D$ and $m \geq 0$ we have $h_{m}(t z, t \bar{z})=t^{m} h_{m}(z, \bar{z})$, so that

$$
\sum_{m=0}^{\infty} h_{m}(z, \bar{z}) t^{m}=\sum_{m=0}^{\infty} h_{m}(t z, t \bar{z})=h(t z, t \bar{z})=0 .
$$

Thus, $h_{m}(z, \bar{z})=0$ and it suffices to prove the lemma for an $m$-homogeneous polynomial on $\mathbb{C} \times \mathbb{C}$.

Suppose $h(z, w)=\sum_{k=0}^{m} a_{k} z^{k} w^{m-k}$ satisfies $h(z, \bar{z})=0$ for all $z \in D$. Taking $0<$ $r<\varrho$ and $\theta \in \mathbb{R}$, and letting $z=r e^{i \theta}$, we easily obtain $\sum_{k=0}^{m} a_{k} e^{2 i k \theta}=0$, for all $\theta \in \mathbb{R}$. Since the functions $e^{2 k i \theta}$, for $k=0,1, \ldots, m$, are linearly independent, we conclude that $a_{0}=a_{1}=\ldots=a_{m}=0$. Hence $h=0$. 
Proof of Theorem 2.2. The function $h: \Omega \times \Omega^{*} \rightarrow \mathbb{C}$ defined by $h(z, w)=$ $\left\langle S K_{\bar{w}}, K_{z}\right\rangle$ for $z, w \in \Omega$ is analytic on $\Omega \times \Omega^{*}$. To show this, writing

$$
h(z, w)=\int_{\Omega}\left(S K_{\bar{w}}\right)(u) \overline{K_{z}(u)} d \mu(u)=\int_{\Omega}\left(S K_{\bar{w}}\right)(u) K_{u}(z) d \mu(u),
$$

we see that $h$ is analytic in the first variable (that is, for fixed $w \in \Omega^{*}$ the function $z \mapsto h(z, w)$ is analytic on $\Omega)$, and using that

$$
h(z, w)=\left\langle K_{\bar{w}}, S^{*} K_{z}\right\rangle=\overline{\left\langle S^{*} K_{z}, K_{\bar{w}}\right\rangle},
$$

we see that $h$ is analytic in the second variable (using the elementary fact that for an analytic function $g$ on $\Omega$ the function $w \mapsto \overline{g(\bar{w})}$ is analytic on $\left.\Omega^{*}\right)$. The mapping $h$ satisfies $h(z, \bar{z})=0$ for all $z \in \Omega$. By Lemma $2.3, h=0$ on $\Omega \times \Omega^{*}$. This means that $\left\langle S K_{w}, K_{z}\right\rangle=0$, for $z, w \in \Omega$, that is, $\left(S K_{w}\right)(z)=0$, for all $z, w \in \Omega$, and hence $S K_{w}=0$ for $w \in \Omega$. It follows that for arbitrary $f \in \mathcal{H}$ and $w \in \Omega$ we have

$$
S^{*} f(w)=\left\langle S^{*} f, K_{w}\right\rangle=\left\langle f, S K_{w}\right\rangle=0 .
$$

Thus $S^{*}=0$, and therefore $S=0$.

Proposition 2.4. Let $\mathcal{H}$ be a reproducing functional Hilbert space of analytic functions on $\Omega$, and suppose that $\mathcal{D}$ is a dense subset of $\mathcal{H}$ such that $\left\langle f, k_{w}\right\rangle \rightarrow 0$ as $w \rightarrow \partial \Omega$ for all $f \in \mathcal{D}$. Then $k_{w} \rightarrow 0$ weakly in $\mathcal{H}$ as $w \rightarrow \partial \Omega$.

Proof. We must show that $\left\langle f, k_{w}\right\rangle \rightarrow 0$ as $w \rightarrow \partial \Omega$, for every $f \in \mathcal{H}$. Given $f \in \mathcal{H}$ and $\varepsilon>0$ choose $h \in \mathcal{D}$ such that $\|f-h\|<\varepsilon$. Then $\left|\left\langle f, k_{w}\right\rangle\right| \leq\left|\left\langle f-h, k_{w}\right\rangle\right|+\left|\left\langle h, k_{w}\right\rangle\right| \leq$ $\|f-h\|+\left|\left\langle h, k_{w}\right\rangle\right|$, so that $\limsup _{w \rightarrow \partial \Omega}\left|\left\langle f, k_{w}\right\rangle\right| \leq\|f-h\|<\varepsilon$, for all $\varepsilon>0$, and thus $\left\langle f, k_{w}\right\rangle \rightarrow 0$ as $w \rightarrow \partial \Omega$

COROLlary 2.5. In each of the spaces $H^{2}, L_{a}^{2}$ and $\mathcal{F}$ we have $k_{w} \rightarrow 0$ weakly as $w \rightarrow \partial \Omega$.

Proof. The space $H^{\infty}$ is dense in both $H^{2}$ and $L_{a}^{2}$, and for an $h \in H^{\infty}$ we have $\left\langle h, k_{w}\right\rangle=h(w) / K_{w}(w) \rightarrow 0$ as $|w| \rightarrow 1^{-}$.

To prove the statement for $\mathcal{F}$, note that $\mathcal{P}$, the space of complex polynomials, is dense in $\mathcal{F}$, and for each fixed $p \in \mathcal{P}$ we have $\left\langle p, k_{w}\right\rangle=p(w) / K_{w}(w)=p(w) e^{-|w|^{2} / 2} \rightarrow 0$ as $|w| \rightarrow \infty$.

Now, if the operator $S$ is compact, then $\widetilde{S}(w) \rightarrow 0$ as $w \rightarrow \partial \Omega$. This leads to the following question. If $S$ is a bounded linear operator for which $\widetilde{S}(w) \rightarrow 0$ as $w \rightarrow \partial \Omega$, is $S$ necessarily compact?

As we will see in the next section, the answer to the above question is "No" in general. The following question seems open.

QUESTION 2.6. For which class of bounded linear operators $S$ on $\mathcal{H}$ is it true that: $S$ is compact if and only if $\widetilde{S}(w) \rightarrow 0$ as $w \rightarrow \partial \Omega$ ?

In Sections 4, 5 and 6 we will give examples of classes of operators for which compactness is characterized by the vanishing condition of the Berezin transform.

Another useful result concerning the Berezin transform is the following proposition. 
Proposition 2.7. Let $\mathcal{H}$ be a reproducing Hilbert space of analytic functions on $\Omega$. If $S$ is a positive or trace-class operator on $\mathcal{H}$, then

$$
\operatorname{tr}(S)=\int_{\Omega} \widetilde{S}(z) K(z, z) d \mu(z)
$$

A proof of the above proposition can be found in [28]. For other properties of the Berezin transform we refer the reader to [6], [14], [17] and [28].

3. Berezin transform and non-compact operators. In this section we will give examples of non-compact operators that have vanishing Berezin transform.

The first example was discovered by Rosenthal [18] in the Hardy space setting. For an analytic function $\varphi: \mathbb{D} \rightarrow \mathbb{D}$ let $C_{\varphi}$ denote the composition operator on $H^{2}$ defined by

$$
C_{\varphi} f=f \circ \varphi, \quad \text { for } f \in H^{2} .
$$

That $C_{\varphi}$ is bounded on $H^{2}$ is well-known. Compactness of $C_{\varphi}$ has been characterized by Shapiro [21], but we will not need his results here. It is easy to compute the Berezin symbol of the operator $C_{\varphi}$. Using (1.5) and the reproducing property of $K_{w}$ we have

$$
\left\langle C_{\varphi} K_{w}, K_{w}\right\rangle=\left(C_{\varphi} K_{w}\right)(w)=K_{w}(\varphi(w))=\frac{1}{1-\bar{w} \varphi(w)} .
$$

Since $\left\|K_{w}\right\|_{2}^{2}=\left\langle K_{w}, K_{w}\right\rangle=K_{w}(w)=1 /\left(1-|w|^{2}\right)$, it follows that

$$
\widetilde{C}_{\varphi}(w)=\frac{1-|w|^{2}}{1-\bar{w} \varphi(w)} \text {. }
$$

Now, let $\psi$ be any conformal map of $\mathbb{D}$ onto the region $R=\{z \in \mathbb{D}: \operatorname{Re} z<0\}$ (such a map $\psi$ exists by the Riemann Mapping Theorem) and let $\varphi(z)=z \psi(z)$, for $z \in \mathbb{D}$. With this notation, Rosenthal [18] obtained the following result.

THEOREM 3.1. The operator $C_{\varphi}$ is not compact on $H^{2}$, while $\widetilde{C}_{\varphi}(w) \rightarrow 0$ as $|w| \rightarrow 1^{-}$.

Proof. Clearly, $|1-\psi(w)| \geq 1$ for all $w \in \mathbb{D}$, and thus $|1-\bar{w} \varphi(w)|=\left.|1-| w\right|^{2} \psi(w) \mid \geq$ $1-\left(1-|w|^{2}\right) \geq 1 / 2$, for $w \in \mathbb{D}$ with $\left(1-|w|^{2}\right)<1 / 2$. For such $w$ 's we have

$$
\left|\widetilde{C}_{\varphi}(w)\right|=\frac{1-|w|^{2}}{|1-\bar{w} \varphi(w)|} \leq 2\left(1-|w|^{2}\right)
$$

and thus $\widetilde{C}_{\varphi}(w) \rightarrow 0$ as $|w| \rightarrow 1^{-}$.

To show that $C_{\varphi}$ is not compact, noting that $z^{n} \rightarrow 0$ weakly in $H^{2}$ it suffices to show that $\left\|C_{\varphi} z^{n}\right\|_{2} \not \rightarrow 0$ as $n \rightarrow \infty$. Observe that $C_{\varphi} z^{n}=\varphi^{n}$. Thus

$$
\left\|C_{\varphi} z^{n}\right\|_{2}^{2}=\int_{0}^{2 \pi}\left|\varphi\left(e^{i \theta}\right)\right|^{2 n} d \theta / 2 \pi=\int_{0}^{2 \pi}\left|\psi\left(e^{i \theta}\right)\right|^{2 n} d \theta / 2 \pi \geq a>0,
$$

since $\left|\psi\left(e^{i \theta}\right)\right|=1$ on a set of positive measure.

The next example is due to Axler [4]. For an analytic function $f$ on $\mathbb{D}$ let $\check{f}(n)$ denote the $n$th Taylor coefficient of $f$, so that $f$ has the representation

$$
f(z)=\sum_{n=0}^{\infty} \check{f}(n) z^{n}, \quad \text { for } z \in \mathbb{D} .
$$

In [4], Axler proved the following theorem. 
Theorem 3.2. The operator $T$ defined on $L_{a}^{2}$ by

$$
T f(z)=\sum_{n=0}^{\infty} \check{f}\left(2^{n}\right) z^{\left(2^{n}\right)}
$$

is a bounded, positive, non-compact operator on $L_{a}^{2}$ such that

$$
\widetilde{T}(w) \rightarrow 0 \quad \text { as }|w| \rightarrow 1^{-} .
$$

Proof. The operator $T$ is clearly bounded, positive, and non-compact. Its Berezin transform is easy to compute: it follows from (1.7) that

$$
T K_{w}(z)=\sum_{n=0}^{\infty}\left(2^{n}+1\right) \bar{w}^{\left(2^{n}\right)} z^{\left(2^{n}\right)}
$$

Thus, using the reproducing property of $K_{w}$, we have

$$
\left\langle T K_{w}, K_{w}\right\rangle=T K_{w}(w)=\sum_{n=0}^{\infty}\left(2^{n}+1\right)\left(|w|^{2}\right)^{\left(2^{n}\right)} .
$$

Since $\left\|K_{w}\right\|^{2}=\left\langle K_{w}, K_{w}\right\rangle=1 /\left(1-|w|^{2}\right)^{2}$, we get

$$
\widetilde{T}(w)=\left(1-|w|^{2}\right)^{2} \sum_{n=0}^{\infty}\left(2^{n}+1\right)\left(|w|^{2}\right)^{\left(2^{n}\right)} .
$$

So, to complete the proof we must show that

$$
(1-x)^{2} \sum_{n=0}^{\infty}\left(2^{n}+1\right) x^{\left(2^{n}\right)} \rightarrow 0 \quad \text { as } x \rightarrow 1 .
$$

This can be done as follows: for fixed $n \in \mathbb{N}$ we have

$$
2^{n-1} x^{\left(2^{n}\right)}=\sum_{k=2^{n-1}+1}^{2^{n}} x^{\left(2^{n}\right)} \leq \sum_{k=2^{n-1}+1}^{2^{n}} x^{k},
$$

and, using the fact that $2^{n}+1 \leq 3 \cdot 2^{n-1}$ for all $n \geq 1$, it follows that

$$
\sum_{n=0}^{\infty}\left(2^{n}+1\right) x^{\left(2^{n}\right)} \leq 2 x+3 \sum_{n=1}^{\infty} \sum_{k=2^{n-1}+1}^{2^{n}} x^{k} \leq 3 \sum_{k=1}^{\infty} x^{k}=3 x /(1-x),
$$

and the statement follows.

With the same notation for entire functions as in the previous example we also have the following result.

Theorem 3.3. The operator $T$ defined on $\mathcal{F}$ by

$$
T f=\sum_{n=0}^{\infty} \check{f}\left(2^{n}\right) z^{\left(2^{n}\right)}
$$

is a bounded, positive, non-compact operator on $\mathcal{F}$ such that

$$
\widetilde{T}(w) \rightarrow 0 \quad \text { as }|w| \rightarrow \infty .
$$

Pr o of. Again $T$ is clearly bounded, positive, and non-compact. Its Berezin transform is again easy to compute:

$$
\widetilde{T}(w)=e^{-|w|^{2} / 2} \sum_{n=0}^{\infty} \frac{1}{\left(2^{n}\right) !}\left(|w|^{2} / 2\right)^{\left(2^{n}\right)} .
$$


We need to show that

$$
e^{-x} \sum_{n=0}^{\infty} \frac{x^{\left(2^{n}\right)}}{\left(2^{n}\right) !} \rightarrow 0 \quad \text { as } x \rightarrow \infty
$$

To prove this statement, we will first use induction to establish that

$$
\lim _{x \rightarrow \infty} e^{-x} \sum_{n=0}^{\infty} \frac{x^{m n}}{(m n) !}=\frac{1}{m},
$$

for $m=1,2,2^{2}, 2^{3}, \ldots$ The above statement is clearly true for $m=1$. To prove the induction step we observe that

$$
\sum_{n=0}^{\infty} \frac{x^{2 m n}}{(2 m n) !}=\frac{1}{2} \sum_{n=0}^{\infty} \frac{x^{m n}}{(m n) !}+\frac{1}{2} \sum_{n=0}^{\infty}(-1)^{n} \frac{x^{m n}}{(m n) !}
$$

and the induction step will be verified once we know that

$$
\lim _{x \rightarrow \infty} e^{-x} \sum_{n=0}^{\infty}(-1)^{n} \frac{x^{m n}}{(m n) !}=0,
$$

for $m=1,2, \ldots$ For $m=1$ this is clearly true. Assuming the above statement, repeatedly applying L'Hospital's rule we arrive at

$$
\lim _{x \rightarrow \infty} e^{-x} \sum_{n=0}^{\infty}(-1)^{n} \frac{x^{m n+m}}{(m n+m) !}=\lim _{x \rightarrow \infty} e^{-x} \sum_{n=0}^{\infty}(-1)^{n} \frac{x^{m n}}{(m n) !}=0
$$

completing the proof of (3.6). To prove (3.4) we reason as follows. Fix $N \in \mathbb{N}$ and put $m=2^{N}$. Then

$$
\sum_{n=N}^{\infty} \frac{x^{\left(2^{n}\right)}}{\left(2^{n}\right) !}=\sum_{k=0}^{\infty} \frac{x^{\left(m 2^{k}\right)}}{\left(m 2^{k}\right) !} \leq \sum_{n=0}^{\infty} \frac{x^{m n}}{(m n) !}
$$

so that

$$
\limsup _{x \rightarrow \infty} e^{-x} \sum_{n=0}^{\infty} \frac{x^{\left(2^{n}\right)}}{\left(2^{n}\right) !}=\limsup _{x \rightarrow \infty} e^{-x} \sum_{n=N}^{\infty} \frac{x^{\left(2^{n}\right)}}{\left(2^{n}\right) !} \leq \lim _{x \rightarrow \infty} e^{-x} \sum_{n=0}^{\infty} \frac{x^{m n}}{(m n) !}=\frac{1}{m}=\frac{1}{2^{N}} .
$$

Since the above inequality holds for all $N \in \mathbb{N},(3.4)$ must hold.

4. Commutativity of Toeplitz operators. Let $\mathcal{H}$ be a reproducing functional Hilbert space of analytic functions on $\Omega$. Then $\mathcal{H}$ is a closed linear subspace of $L^{2}(\Omega, \mu)$ so that there exists an orthogonal projection $P$ of $L^{2}(\Omega, \mu)$ onto $\mathcal{H}$. This projection is easily expressed in terms of the reproducing kernel $K$ :

$$
P f(w)=\left\langle P f, K_{w}\right\rangle=\left\langle f, K_{w}\right\rangle=\int_{\Omega} f(z) \overline{K_{w}(z)} d \mu(z)=\int_{\Omega} f(z) K(w, z) d \mu(z) .
$$

If a bounded $f$ is in $L^{2}(\Omega, \mu)$, we define the Toeplitz operator $T_{f}: \mathcal{H} \rightarrow \mathcal{H}$ by $T_{f}(g)=$ $P(f g)$, for $g \in \mathcal{H}$.

Toeplitz operators on the Hardy space $H^{2}$ are easily recognized by their matrix with respect to the standard orthonormal basis: for $f \in L^{\infty}(\mathbb{T})$ we have $\left\langle T_{f} z^{n}, z^{m}\right\rangle=$ $\left\langle f z^{n}, z^{m}\right\rangle=\int_{0}^{2 \pi} f\left(e^{i \theta}\right) e^{i(n-m)} d \theta / 2 \pi$, for $n, m \geq 0$, so the matrix of $T_{f}$ with respect to the standard basis is a Toeplitz matrix. 
EXAMPLE 4.1. It follows from (1.5) and (1.3) that in $H^{2}$ the normalized reproducing kernel $k_{w}$ is given by $k_{w}(z)=K_{w}(z) /\left\|K_{w}\right\|=\left(1-|w|^{2}\right)^{1 / 2} /(1-\bar{w} z)$. Thus, for $f \in L^{\infty}(\mathbb{T})$ we have

$$
\begin{aligned}
\widetilde{T}_{f}(w)=\left\langle f k_{w}, k_{w}\right\rangle & =\lim _{r \rightarrow 1^{-}} \int_{0}^{2 \pi} f\left(r e^{i \theta}\right) \frac{1-|w|^{2}}{\left|1-r e^{-i \theta} w\right|^{2}} \frac{d \theta}{2 \pi} \\
& =\int_{0}^{2 \pi} f\left(e^{i \theta}\right) \frac{1-|w|^{2}}{\left|e^{i \theta}-w\right|^{2}} \frac{d \theta}{2 \pi}=\hat{f}(w),
\end{aligned}
$$

the Poisson extension of $f$ at $w$.

The following result is classical. We will give a very short proof using Theorem 2.2.

Theorem 4.2. Let $f \in L^{\infty}(\mathbb{T})$. Then: the Toeplitz operator $T_{f}$ is compact on $H^{2} \Leftrightarrow$ $f=0$ a.e. on $\mathbb{T}$.

Proof. If $T_{f}$ is compact, then $\widetilde{f}(w)=\widetilde{T}_{f}(w) \rightarrow 0$ as $|w| \rightarrow 1^{-}$. But $\tilde{f}=\hat{f}$, the Poisson extension of $f$ over $\mathbb{D}$, so that $\widetilde{f}(r \zeta) \rightarrow f(\zeta)$ as $r \rightarrow 1^{-}$, for a.e. $\zeta \in \mathbb{T}$, and we conclude that $f(\zeta)=0$ for a.e. $\zeta \in \mathbb{T}$.

As another example of Theorem 2.2 we will prove the following result of Brown and Halmos [11], whose original proof was based on matrices. We first derive a preliminary result. If $\mathcal{H}$ is a reproducing functional Hilbert space of functions on the set $\Omega$, and $h$ is a function on $\Omega$ such that $h K_{z} \in \mathcal{H}$ for all $z \in \Omega$, then $P\left(\bar{h} K_{w}\right)$ is easily determined as follows:

$$
\begin{aligned}
P\left(\bar{h} K_{w}\right)(z) & =\left\langle P\left(\bar{h} K_{w}\right), K_{z}\right\rangle=\left\langle\bar{h} K_{w}, K_{z}\right\rangle=\left\langle K_{w}, h K_{z}\right\rangle \\
& =\overline{\left\langle h K_{z}, K_{w}\right\rangle}=\overline{h(w) K_{z}(w)}=\overline{h(w)} K_{w}(z),
\end{aligned}
$$

so that

$$
P\left(\bar{h} k_{w}\right)=\overline{h(w)} k_{w} .
$$

Note that if $\mathcal{H}$ is $H^{2}$ or $L_{a}^{2}$ the reproducing kernels are in fact bounded, so that the above formula holds for any function $h \in \mathcal{H}$ and $w \in \mathbb{D}$.

The following theorem is due to Brown and Halmos [11]:

TheOREM 4.3. If $f$ and $g$ in $L^{\infty}(\mathbb{T})$ are such that $T_{f}$ and $T_{g}$ commute on $H^{2}$, then:

(i) both $f$ and $g$ are analytic, or

(ii) both $f$ and $g$ are co-analytic, or

(iii) a non-trivial linear combination of $f$ and $g$ is constant.

Proof. Compute the Berezin transform of the commutator $\left[T_{f}, T_{g}\right]$ :

$$
\left.\widetilde{\left[T_{f}, T_{g}\right.}\right](w)=\left\langle T_{f} T_{g} k_{w}, k_{w}\right\rangle-\left\langle T_{g} T_{f} k_{w}, k_{w}\right\rangle=\left\langle T_{g} k_{w}, \bar{f} k_{w}\right\rangle-\left\langle T_{f} k_{w}, \bar{g} k_{w}\right\rangle .
$$

Write $f=f_{1}+\bar{f}_{2}$ and $g=g_{1}+\bar{g}_{2}$, where $f_{1}, f_{2}, g_{1}$ and $g_{2}$ are analytic on $\mathbb{D}$. Then we have $T_{g} k_{w}=P\left(g k_{w}\right)=g_{1} k_{w}+P\left(\bar{g}_{2} k_{w}\right)=g_{1} k_{w}+\overline{g_{2}(w)} k_{w}$. Thus, using the reproducing property of $k_{w}$,

$$
\begin{aligned}
\left\langle T_{g} k_{w}, \bar{f} k_{w}\right\rangle & =\left\langle g_{1} k_{w}+\overline{g_{2}(w)} k_{w}, \bar{f}_{1} k_{w}+f_{2} k_{w}\right\rangle \\
& =f_{1}(w) g_{1}(w)+f_{1}(w) \overline{g_{2}(w)}+\left\langle\bar{f}_{2} g_{1} k_{w}, k_{w}\right\rangle+\overline{f_{2}(w) g_{2}(w)}
\end{aligned}
$$


and, by interchanging the roles of $f$ and $g$, we also have

$$
\left\langle T_{f} k_{w}, \bar{g} k_{w}\right\rangle=f_{1}(w) g_{1}(w)+g_{1}(w) \overline{f_{2}(w)}+\left\langle f_{1} \bar{g}_{2} k_{w}, k_{w}\right\rangle+\overline{f_{2}(w) g_{2}(w)} .
$$

Hence

$$
\left[\widetilde{T_{f}, T_{g}}\right]=\widetilde{u}-u
$$

where $u=\bar{f}_{2} g_{1}-f_{1} \bar{g}_{2}$. Thus, $T_{f}$ and $T_{g}$ commute if and only if $\left[\widetilde{T_{f}, T_{g}}\right] \equiv 0$, if and only if $\widetilde{u}=u$, that is, if and only if $u=\bar{f}_{2} g_{1}-f_{1} \bar{g}_{2}$ is harmonic on $\mathbb{D}$. This is equivalent to $4\left(\bar{f}_{2}^{\prime} g_{1}^{\prime}-f_{1}^{\prime} \bar{g}_{2}^{\prime}\right)=\Delta u \equiv 0$, which is easily seen to be equivalent to one of conditions (i), (ii) or (iii).

Combining (1.8) and (1.3) we see that in $L_{a}^{2}$ the normalized reproducing kernel $k_{w}$ is given by $k_{w}(z)=K_{w}(z) /\left\|K_{w}\right\|=\left(1-|w|^{2}\right) /(1-\bar{w} z)^{2}$. Thus, in the Bergman setting we have the following formula for the Berezin symbol of a function: for $f \in L^{\infty}(\mathbb{D})$ and $w \in \mathbb{D}$ we have

$$
\widetilde{f}(w)=\widetilde{T_{f}}(w)=\int_{\mathbb{D}} f(z) \frac{\left(1-|w|^{2}\right)^{2}}{|1-\bar{w} z|^{4}} d A(z) .
$$

The above argument would also work on the Bergman space, provided the equation $\widetilde{u}=u$ implies that $u$ must be harmonic on $\mathbb{D}$. Although this has recently been shown by Ahern, Florin and Rudin [1], we will follow the approach of Axler and Cučković [5] who obtained the analogue of Theorem 4.3 in the Bergman setting. Their main tool is an invariant mean value characterization for harmonic functions. We will need to introduce more notation before we can state their result.

We will first discuss the Möbius-invariance of the Berezin transform. For $w \in \mathbb{D}$ define the fractional transformation $\varphi_{w}: \mathbb{D} \rightarrow \mathbb{D}$ by

$$
\varphi_{w}(z)=\frac{w-z}{1-\bar{w} z}, \quad z \in \mathbb{D} .
$$

It is easily seen that $\varphi_{w}$ is its own inverse: $\varphi_{w}\left(\varphi_{w}(z)\right)=z$ for all $z \in \mathbb{D}$. The function $\varphi_{w}$ has derivative given by $\varphi_{w}^{\prime}(z)=\left(|w|^{2}-1\right) /(1-\bar{w} z)^{2}$, so the change-of-variable $u=\varphi_{w}(z)$ has real Jacobian equal to $\varphi_{w}^{\prime}(z) \overline{\varphi_{w}^{\prime}(z)}=\left|\varphi_{w}^{\prime}(z)\right|^{2}=\left(1-|w|^{2}\right)^{2} /|1-\bar{w} z|^{4}$. It follows that $\widetilde{f}(w)$ is also given by the following formula:

$$
\widetilde{f}(w)=\int_{\mathbb{D}}\left(f \circ \varphi_{w}\right)(z) d A(z) .
$$

It is this last formula that shows the following invariance of the Berezin symbol:

$$
\widetilde{f}(\varphi(w))=(f \circ \varphi)^{\sim}(w),
$$

for all $\varphi \in \operatorname{Aut}(\mathbb{D})$. Our approach will exploit the Möbius-invariance of the Berezin transform.

Before we can state Axler and Cučković's invariant mean value characterization for harmonic functions, we need another definition. For a function $v$ on $\mathbb{D}$ define its radialization $\mathcal{R}(v)$ by

$$
\mathcal{R}(v)(z)=\int_{0}^{2 \pi} v\left(e^{i \theta} z\right) \frac{d \theta}{2 \pi}, \quad z \in \mathbb{D} .
$$

Note that $\mathcal{R}(v)$ is a radial function on $\mathbb{D}: \mathcal{R}(v)(z)$ only depends on $|z|$. 
The following lemma is due to Axler and Čučković [5].

Lemma 4.5. If $u \in L^{1}(\mathbb{D}, d A)$ is such that $\widetilde{u}=u$ and for each $w \in \mathbb{D}$ the function $\mathcal{R}\left(u \circ \varphi_{w}\right)$ extends to a continuous function on $\overline{\mathbb{D}}$, then $u$ is harmonic on $\mathbb{D}$.

The following result is needed in the proving the above lemma (see [2] or [19]). For completeness we include a proof.

Proposition 4.6. If $v$ is continuous on $\overline{\mathbb{D}}$, and $\widetilde{v}=v$, then $v$ is harmonic on $\mathbb{D}$.

Proof. We can without loss of generality assume that $v$ is real-valued. Writing $\hat{v}$ for the Poisson integral of the restriction of $v$ to $\mathbb{T}$, the function $h:=v-\hat{v}$ satisfies $\widetilde{h}=h$ and $h \equiv 0$ on $\mathbb{T}$. If $h$ takes on its maximum value at the point $w \in \mathbb{D}$, then

$$
h(w)=\widetilde{h}(w)=\int_{\mathbb{D}} h\left(\varphi_{w}(z)\right) d A(z) \leq h(w),
$$

implies that $h\left(\varphi_{w}(z)\right)=h(w)$ for all $z \in \mathbb{D}$, and consequently, $h$ must be constant. We conclude that $h$ takes on its maximum value at a point of $\mathbb{T}$. Because $h \equiv 0$ on $\mathbb{T}$ we have $h \leq 0$. But also $-h \leq 0$, and thus $h \equiv 0$. Hence $v=\hat{v}$ is harmonic on $\mathbb{D}$.

The following proposition shows that the Berezin transform and radialization commute.

Proposition 4.7. If $u \in L^{1}(\mathbb{D}, d A)$, then

$$
\widetilde{\mathcal{R}(u)}=\mathcal{R}(\widetilde{u})
$$

Pr o o f. Using equation (4.4), the definition of the radialization and Fubini's theorem, we have

$$
\begin{aligned}
\widetilde{\mathcal{R}(u)}(w) & =\int_{\mathbb{D}} \mathcal{R}(u)\left(\varphi_{w}(z)\right) d A(z)=\int_{\mathbb{D}}^{2 \pi} u\left(e_{0}^{i \theta} \varphi_{w}(z)\right) \frac{d \theta}{2 \pi} d A(z) \\
& =\int_{0}^{2 \pi} \int_{\mathbb{D}} u\left(\varphi_{e^{i \theta} w}\left(e^{i \theta} z\right)\right) d A(z) \frac{d \theta}{2 \pi}=\int_{0}^{2 \pi} \int_{\mathbb{D}} u\left(\varphi_{e^{i \theta} w}(z)\right) d A(z) \frac{d \theta}{2 \pi} \\
& =\int_{0}^{2 \pi} \widetilde{u}\left(e^{i \theta} w\right) \frac{d \theta}{2 \pi}=\mathcal{R}(\widetilde{u})(w),
\end{aligned}
$$

where the use of Fubini's theorem is justified by replacing each occurrence of $u$ by $|u|$.

One more preliminary result remains. The following is implicit in Axler and Čučković [5].

Lemma 4.8. If $f, g \in H^{2}$, then $\mathcal{R}(f \bar{g})$ extends continuously to $\overline{\mathbb{D}}$.

Proof. Writing $f(z)=\sum_{n=0}^{\infty} a_{n} z^{n}$ and $g(z)=\sum_{n=0}^{\infty} b_{n} z^{n}$, it is easily seen that

and since

$$
\mathcal{R}(f \bar{g})(z)=\sum_{n=0}^{\infty} a_{n} \bar{b}_{n}|z|^{2 n}
$$

the stated result follows.

$$
\sum_{n=0}^{\infty}\left|a_{n} \bar{b}_{n}\right| \leq\left(\sum_{n=0}^{\infty}\left|a_{n}\right|^{2}\right)^{1 / 2}\left(\sum_{n=0}^{\infty}\left|b_{n}\right|^{2}\right)^{1 / 2}<\infty,
$$


Proof of Lemma 4.5. If $\widetilde{u}=u$, then $\widetilde{\mathcal{R}(u)}=\mathcal{R}(u)$. Since $v=\mathcal{R}(u)$ extends to a continuous function on $\overline{\mathbb{D}}$, the function $v$ must be harmonic, and thus constant (since it is radial). Using Taylor's theorem it is easy to verify that

$$
\Delta u(0)=\lim _{r \rightarrow 0^{+}} \frac{4}{r^{2}} \int_{0}^{2 \pi}\left(u\left(r e^{i \theta}\right)-u(0)\right) \frac{d \theta}{2 \pi}=\lim _{r \rightarrow 0^{+}} \frac{4}{r^{2}}(\mathcal{R}(u)(r)-\mathcal{R}(u)(0))=0 .
$$

Now, for $w \in \mathbb{D}$ we also have $\left(u \circ \varphi_{w}\right)^{\sim}=u \circ \varphi_{w}$, and applying the above argument to $u \circ \varphi_{w}$ we obtain $\Delta\left(u \circ \varphi_{w}\right)(0)=0$. It is easy to verify that $\Delta\left(u \circ \varphi_{w}\right)(0)=(1-$ $\left.|w|^{2}\right)^{2} \Delta u(w)$, thus $\Delta u(w)=0$.

We are now ready to prove the following result of Axler and Čučković [5]:

THEOREM 4.9. Let $f$ and $g$ be bounded harmonic functions on $\mathbb{D}$. Then: $T_{f}$ and $T_{g}$ commute on $L_{a}^{2}$ if and only if:

(i) both $f$ and $g$ are analytic, or

(ii) both $f$ and $g$ are co-analytic, or

(iii) a non-trivial linear combination of $f$ and $g$ is constant.

Proof. Write $f=f_{1}+\bar{f}_{2}$ and $g=g_{1}+\bar{g}_{2}$, where $f_{1}, f_{2}, g_{1}$ and $g_{2}$ are analytic functions on $\mathbb{D}$ and assume that $T_{f}$ and $T_{g}$ commute. As in the proof of Theorem 4.3 we have $\widetilde{u}=u$, where $u=\bar{f}_{2} g_{1}-f_{1} \bar{g}_{2}$. The functions $f_{1}, f_{2}, g_{1}$ and $g_{2}$ need not be bounded, however, the boundedness of $f$ and $g$ implies that $f_{1}, f_{2}, g_{1}$ and $g_{2}$ must belong to $H^{2}$. To see this, use the fact that

$$
\int_{0}^{2 \pi}\left|f_{1}\left(r e^{i \theta}\right)-f_{1}(0)\right|^{2} \frac{d \theta}{2 \pi}+\int_{0}^{2 \pi}\left|f_{2}\left(r e^{i \theta}\right)-f_{2}(0)\right|^{2} \frac{d \theta}{2 \pi}=\int_{0}^{2 \pi}\left|f\left(r e^{i \theta}\right)-f(0)\right|^{2} \frac{d \theta}{2 \pi},
$$

for all $0<r<1$. It follows that $\mathcal{R}(u)$ extends continuously to $\overline{\mathbb{D}}$. Replacing $u$ by $u \circ \varphi_{w}$ we see that the conditions of Lemma 4.5 are satisfied, and hence $u$ is harmonic on $\mathbb{D}$. This easily implies that one of conditions (i), (ii) and (iii) must hold, and proves the necessity of (i), (ii) or (iii). The sufficiency is easily established.

In [25] the author has shown that essential commutativity of Toeplitz operators with harmonic symbols on $L_{a}^{2}$ is characterized by local versions of statements (i), (ii) or (iii). More precisely, we have the following result, where we refer the reader to [25] for some of the missing terminology.

THEOREM 4.10. Let $f$ and $g$ be bounded harmonic functions on $\mathbb{D}$. Then the following statements are equivalent:

(a) $T_{f}$ and $T_{g}$ are essentially commuting on $L_{a}^{2}$ (i.e., the commutator $\left[T_{f}, T_{g}\right]$ is compact on $\left.L_{a}^{2}\right)$;

(b) on each Gleason part $G$ of the maximal ideal space of $H^{\infty}$, except possibly $\mathbb{D}$ :

(i) both $f$ and $g$ are analytic on $G$, or

(ii) both $f$ and $g$ are co-analytic on $G$, or

(iii) a non-trivial linear combination of $f$ and $g$ is constant on $G$;

(c) $\lim _{|z| \rightarrow 1^{-}}\left(1-|z|^{2}\right)^{2}\left\{\frac{\partial f}{\partial \bar{z}} \frac{\partial g}{\partial z}-\frac{\partial f}{\partial z} \frac{\partial g}{\partial \bar{z}}\right\}=0$. 
Using the same notation as in the proof of Theorem 4.9, we note that condition (c) of Theorem 4.10 says that $\widetilde{u}(z)-u(z) \rightarrow 0$ as $|z| \rightarrow 1^{-}$, so that the compactness of $\left[T_{f}, T_{g}\right]$ is equivalent with the condition $\left[T_{f}, T_{g}\right] \sim(z) \rightarrow 0$ as $|z| \rightarrow 1^{-}$.

Condition (b) of Theorem 4.10 gives various possibilities for the behavior on each of the Gleason parts other than $\mathbb{D}$. It is an open question whether for non-constant bounded harmonic functions on $\mathbb{D}$ satisfying condition (b) the behavior can be different on distinct Gleason parts other than $\mathbb{D}$.

5. Berezin transform and Hankel and Toeplitz operators. In this section we will discuss several positive results relating certain properties of classes of operators to the boundary behavior of the Berezin transform. We start in the setting of the Fock space. By (1.10) and (1.3) we have: $k_{w}(z)=K_{w}(z) /\left\|K_{w}\right\|=e^{\bar{w} z / 2} / e^{|w|^{2} / 4}=e^{\bar{w} z / 2-|w|^{2} / 4}$. Thus the Berezin transform of a Toeplitz operator $T_{f}$ on $\mathcal{F}$ is given by

$$
\widetilde{f}(w)=\widetilde{T}_{f}(w)=\int_{\mathbb{C}} f(z)\left|e^{\bar{w} z / 2-|w|^{2} / 4}\right|^{2} d \mu(z)=\int_{\mathbb{C}} f(z) e^{-|z-w|^{2} / 2} d A(z) / 2 .
$$

The map $f \mapsto \tilde{f}$ is a smoothing operator which is related to the heat equation on $\mathbb{C}=\mathbb{R}^{2}$. In fact,

$$
\widetilde{f}(t, w)=\frac{1}{4 t} \int_{\mathbb{C}} f(z) e^{-|z-w|^{2} /(4 t)} d A(z)
$$

is the unique solution to the heat equation with initial value $f$ (at $t=0$ ). Thus $\tilde{f}(w)=$ $\widetilde{f}(1 / 2, w)$ is the solution of the initial value problem for $f$ at $t=1 / 2$. Berger and Coburn ([8], [9]) used this fact in their analysis of Toeplitz operators on $\mathcal{F}$. Using a different method we obtained the following result for Toeplitz operators, and Hankel operators $H_{f}: \mathcal{F} \rightarrow L^{2}(\mathbb{C}, d \mu)$ defined by $H_{f} g=(I-P)(f g)$, for $g \in \mathcal{F}:$

TheOrem 5.1. For $f \in L^{\infty}(\mathbb{C})$ :

(a) $T_{f}$ is compact on $\mathcal{F} \Leftrightarrow\left\|P\left(f \circ \tau_{w}\right)\right\|_{2} \rightarrow 0$ as $|w| \rightarrow \infty$;

(b) $H_{f}$ is compact on $\mathcal{F} \Leftrightarrow\left\|f \circ \tau_{w}-P\left(f \circ \tau_{w}\right)\right\|_{2} \rightarrow 0$ as $|w| \rightarrow \infty$,

where $\tau_{w}: \mathbb{C} \rightarrow \mathbb{C}$ is the translation $\tau_{w}(z)=z+w$.

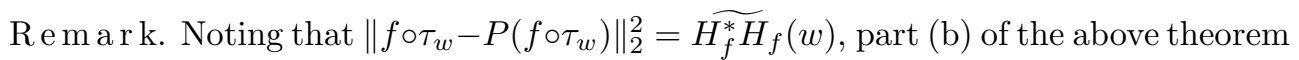
says that the compactness of $H_{f}$, and thus of $H_{f}^{*} H_{f}$, is equivalent to $\widetilde{H_{f}^{*} H_{f}}(w) \rightarrow 0$ as $|w| \rightarrow \infty$.

Writing $M_{f}$ for the multiplication operator $M_{f}: \mathcal{F} \rightarrow L^{2}(\mathbb{C}, d \mu)$ defined by $M_{f} g=f g$, for $g \in \mathcal{F}$, the above theorem easily implies the following result of Berger and Coburn [9]:

THEOREM 5.2. For $f \in L^{\infty}(\mathbb{C})$ :

$$
M_{f} \text { is compact on } \mathcal{F} \Leftrightarrow \widetilde{|f|^{2}}(w) \rightarrow 0 \text { as }|w| \rightarrow \infty \text {. }
$$

Proof. For $w \in \mathbb{C}$ we have

$$
\widetilde{|f|^{2}}(w)=\left\|f \circ \tau_{w}\right\|_{2}^{2}=\left\|P\left(f \circ \tau_{w}\right)\right\|_{2}^{2}+\left\|f \circ \tau_{w}-P\left(f \circ \tau_{w}\right)\right\|_{2}^{2},
$$

so if $\widetilde{|f|^{2}}(w) \rightarrow 0$ as $|w| \rightarrow \infty$, then both $\left\|P\left(f \circ \tau_{w}\right)\right\|_{2} \rightarrow 0$ as $|w| \rightarrow \infty$ and $\| f \circ \tau_{w}-$ $P\left(f \circ \tau_{w}\right) \|_{2} \rightarrow 0$ as $|w| \rightarrow \infty$, and by Theorem 5.1 both $T_{f}$ and $H_{f}$ are compact. Thus $M_{f}$ is compact. 
Note that the above theorem can be used to answer our question about compactness for Toeplitz operators with positive symbols:

Proposition 5.3. If $f$ is a non-negative bounded measurable function on $\mathbb{C}$, then: $T_{f}$ is compact on $\mathcal{F} \Leftrightarrow \tilde{f}(w) \rightarrow 0$ as $|w| \rightarrow \infty$.

Pr o of. The implication " $\Leftarrow$ ": Let $g$ be a non-negative measurable function on $\mathbb{C}$ such that $f=g^{2}$. Then $\mid g^{2}(w)=\widetilde{f}(w) \rightarrow 0$ as $|w| \rightarrow \infty$, and by Theorem $5.2, M_{g}$ is compact. Then also $M_{f}=M_{g}^{2}$ is compact, and hence $T_{f}$ is compact.

Theorem 5.1 also gives some insight in the following result of Berger and Coburn [9], which they proved by an averaging operation over a representation of the Heisenberg group related to the operation of taking the Berezin transform.

THEOREM 5.4. For $f \in L^{\infty}(\mathbb{C})$ the following statements are equivalent:

(i) $H_{f}$ is compact on $\mathcal{F}$;

(ii) $H_{f}$ is compact on $\mathcal{F}$;

(iii) $\left\|f \circ \tau_{w}-\tilde{f}(w)\right\|_{2} \rightarrow 0$ as $|w| \rightarrow \infty$;

(iv) $\overline{|f|^{2}}(w)-|\tilde{f}(w)|^{2} \rightarrow 0$ as $|w| \rightarrow \infty$.

Informal proof. If $\left(w_{n}\right)_{n}$ is a sequence in $\mathbb{C}$ such that $\left|w_{n}\right| \rightarrow \infty$ as $n \rightarrow \infty$, then by going to a subsequence we may assume that $P\left(f \circ \tau_{w_{n}}\right) \rightarrow h$ in $\mathcal{F}$ as $n \rightarrow \infty$ (by compactness of the operator $P$ restricted to $L^{\infty}(\mathbb{C})$, a result that can be proved similarly to Theorem 20 in [9]). If $H_{f}$ is compact, then $\left\|f \circ \tau_{w_{n}}-P\left(f \circ \tau_{w_{n}}\right)\right\|_{2} \rightarrow 0$ as $n \rightarrow \infty$, and thus $\left\|f \circ \tau_{w_{n}}-h\right\|_{2} \rightarrow 0$ as $n \rightarrow \infty$. But, since the $f \circ \tau_{w_{n}}$ are uniformly bounded, this implies that the entire function $h$ must be bounded! Thus $h$ is constant, and it is easily shown that also $\left\|\bar{f} \circ \tau_{w_{n}}-P\left(\bar{f} \circ \tau_{w_{n}}\right)\right\|_{2} \rightarrow 0$ as $n \rightarrow \infty$, so that by Theorem 5.1 the operator $H_{\bar{f}}$ is compact.

On the Bergman space the author and D. Zheng [26] obtained the following result, completely analogous to Theorem 5.1:

TheOREM 5.5. For $f \in L^{\infty}(\mathbb{D})$ :

(a) $T_{f}$ is compact on $L_{a}^{2} \Leftrightarrow\left\|P\left(f \circ \varphi_{w}\right)\right\|_{2} \rightarrow 0$ as $|w| \rightarrow 1^{-}$;

(b) $H_{f}$ is compact on $L_{a}^{2} \Leftrightarrow\left\|f \circ \varphi_{w}-P\left(f \circ \varphi_{w}\right)\right\|_{2} \rightarrow 0$ as $|w| \rightarrow 1^{-}$.

Results analogous to Theorem 5.2 and Proposition 5.3 hold for the Bergman space.

For points $z, w \in \mathbb{D}$ the pseudo-hyperbolic distance between $z$ and $w$ is defined by $d(z, w)=\left|\varphi_{w}(z)\right|$. That $d$ is indeed a metric on $\mathbb{D}$ is non-trivial. Note that $d$ is invariant under the automorphism group of $\mathbb{D}: d(z, w)=d(\varphi(z), \varphi(w))$, for all $\varphi \in \operatorname{Aut}(\mathbb{D})$. The Bergman metric is given by the formula $\varrho(z, w)=\frac{1}{2} \log \frac{1+d(z, w)}{1-d(z, w)}$, for $z, w \in \mathbb{D}$. It is clear that also $\varrho$ is invariant under Aut $(\mathbb{D})$. Békollé, Berger, Coburn and Zhu [7] have shown that the Berezin symbol of a class of measurable functions including the bounded measurable functions satisfies a growth condition that implies that it is Lipschitz with respect to the Bergman metric (see Section 7.1 of [28] for a proof in the setting of the unit disk). In particular, if $g \in L^{\infty}(\mathbb{D})$, then the function $\widetilde{g}$ is Lipschitz with respect to $\varrho$.

The next result partially answers the question in Section 2. In the next section we will give examples for which the Lipschitz condition is not needed. 
TheOREM 5.6. If $f$ is Lipschitz with respect to the Bergman metric on $\mathbb{D}$, then

$$
T_{f} \text { is compact on } L_{a}^{2} \Leftrightarrow \tilde{f}(w) \rightarrow 0 \text { as }|w| \rightarrow 1^{-} .
$$

Proof. The implication " $\Rightarrow$ " is always true, since $\widetilde{f}(z)=\left\langle T_{f} k_{z}, k_{z}\right\rangle$, and $k_{z} \rightarrow 0$ weakly in $L_{a}^{2}$ as $|z| \rightarrow 1^{-}$(by Proposition 2.4).

The implication " $\Leftarrow$ ": By Theorem 5.5 it suffices to prove that $\left\|P\left(f \circ \varphi_{z}\right)\right\|_{2} \rightarrow 0$ as $|z| \rightarrow 1^{-}$. We adopt the same notation as in [26]: we write $\Phi$ for the set of all possible limits in the product space $(\beta \mathbb{D})^{\mathbb{D}}$ of nets $\left(\varphi_{z_{\alpha}}\right)$ for which $\left|z_{\alpha}\right| \rightarrow 1^{-}$. (Here $\beta \mathbb{D}$ denotes the Cech-Stone compactification of $\mathbb{D}$.) Since $f$ is a bounded continuous function on $\mathbb{D}$, it has a unique continuous extension to $\beta \mathbb{D}$, which we will denote by $f^{\beta}$. If $\varphi \in \Phi$ is the limit in $(\beta \mathbb{D})^{\mathbb{D}}$ of a net $\left(\varphi_{z_{\alpha}}\right)$ for which $\left|z_{\alpha}\right| \rightarrow 1^{-}$, then it is easily seen that $f \circ \varphi_{z_{\alpha}} \rightarrow f^{\beta} \circ \varphi$ pointwise on $\mathbb{D}$. We claim that the convergence is uniform on compact subsets of $\mathbb{D}$. To prove this we need only show that the family $\left\{f \circ \varphi_{z}: z \in \mathbb{D}\right\}$ is equicontinuous. But this easily follows from the the Möbius-invariance of the Bergman metric $\varrho$ and the fact that $f$ is uniformly continuous with respect to $\varrho$.

It follows from our claim that $f \circ \varphi_{z_{\alpha}} \rightarrow f^{\beta} \circ \varphi$ in $L^{2}(\mathbb{D})$, and thus

$$
P\left(f \circ \varphi_{z_{\alpha}}\right) \rightarrow P\left(f^{\beta} \circ \varphi\right)
$$

in $L_{a}^{2}$. Since evaluation at 0 is continuous, we conclude that

$$
\widetilde{f}\left(z_{\alpha}\right)=P\left(f \circ \varphi_{z_{\alpha}}\right)(0) \rightarrow P\left(f^{\beta} \circ \varphi\right)(0)=\left(f^{\beta} \circ \varphi\right)^{\sim}(0) .
$$

Now, by assumption, $\widetilde{f}\left(z_{\alpha}\right) \rightarrow 0$, thus $\left(f^{\beta} \circ \varphi\right)^{\sim}(0)=0$.

Now, let $z \in \mathbb{D}$ be fixed. It is a consequence of Schwarz' Lemma that

$$
\left(\varphi_{z_{\alpha}} \circ \varphi_{z}\right)(w)=\varphi_{\varphi_{z_{\alpha}}(z)}\left(c_{\alpha} w\right),
$$

for all $w \in \mathbb{D}$, where $\left|c_{\alpha}\right|=1$ for each $\alpha$. By going to a subnet, which we will not relabel, we may assume that $c_{\alpha} \rightarrow c$ and $\varphi_{\varphi_{z_{\alpha}}(z)} \rightarrow \psi$ in $\Phi$. Then we have

$$
\left(\varphi \circ \varphi_{z}\right)(w)=\psi(c w)
$$

for all $w \in \mathbb{D}$. By the previous paragraph, $\left(f^{\beta} \circ \psi\right)^{\sim}(0)=0$. But,

$$
\left(f^{\beta} \circ \psi\right)^{\sim}(0)=\left(f^{\beta} \circ \varphi \circ \varphi_{z}\right)^{\sim}(0)=\left(f^{\beta} \circ \varphi\right)^{\sim}\left(\varphi_{z}(0)\right)=\left(f^{\beta} \circ \varphi\right)^{\sim}(z),
$$

and thus $\left(f^{\beta} \circ \varphi\right)^{\sim}(z)=0$ for all $z \in \mathbb{D}$. This implies that $T_{f^{\beta} \circ \varphi}=0$ (by Theorem 2.2), so that $P\left(f^{\beta} \circ \varphi\right) \equiv 0$. Hence $P\left(f \circ \varphi_{z_{\alpha}}\right) \rightarrow 0$ in $L_{a}^{2}$, and we conclude that indeed $\left\|P\left(f \circ \varphi_{z}\right)\right\|_{2} \rightarrow 0$ as $|z| \rightarrow 1^{-}$, proving that $T_{f}$ is compact (by part (a) of Theorem 5.5).

We note that the above theorem also holds in the setting of the Fock space:

THEOREM 5.7. If $f$ is a bounded Lipschitz function on $\mathbb{C}$, then

$$
T_{f} \text { is compact on } \mathcal{F} \Leftrightarrow \tilde{f}(w) \rightarrow 0 \text { as }|w| \rightarrow \infty \text {. }
$$

6. Sarason's example. In his survey article [3] Axler mentioned that Sarason had constructed a bounded measurable function $f$ for which $f^{2}$ is identically 1 on $\mathbb{D}$ and $T_{f}$ is compact on $L_{a}^{2}$. Axler gave no specific details in his article; I received the following construction and its proof through correspondence with Sarason [20]. 
Sarason's construction. Let $\left(r_{k}\right)$ and $\left(s_{k}\right)$ be sequences of positive numbers converging to 1 with $0=r_{1}<s_{1}<r_{2}<s_{2}<\ldots$, and define $f$ on $\mathbb{D}$ by

$$
f(x)= \begin{cases}-1 & \text { if } r_{k} \leq|z|<s_{k} \\ 1 & \text { if } s_{k} \leq|z|<r_{k+1}\end{cases}
$$

Then the eigenvalues of $T_{f}$ are given by

$$
\lambda_{n}=(n+1) \int_{\mathbb{D}} f(z)|z|^{2 n} d A(z)=\sum_{k=1}^{\infty}\left(r_{k+1}^{2 n+2}-2 s_{k}^{2 n+2}+r_{k}^{2 n+2}\right) .
$$

The problem is to choose the $r_{k}$ and $s_{k}$ so that $\lambda_{n} \rightarrow 0$ as $n \rightarrow \infty$. By carefully estimating the eigenvalues, Sarason [20] showed that for $r_{k}=1-1 / k^{2 / 3}$ and $s_{k}=\left(r_{k}+r_{k+1}\right) / 2$ the operator $T_{f}$ is compact on $L_{a}^{2}$. Since we are able to prove slightly more we omit Sarason's proof. In fact, we have the following more general result:

THEOREM 6.3. Let $f$ be the function given by (6.1) with $r_{k}=1-1 / k^{\alpha}$, where $\alpha>0$, and $s_{k}=\left(r_{k}+r_{k+1}\right) / 2$. Then

$$
T_{f} \text { is trace-class on } L_{a}^{2} \Leftrightarrow \alpha<1 \text {. }
$$

Proof. By convexity of the function $y=x^{2 n+2}$, the $k$ th summand in the sum of $\lambda_{n}$ is non-negative, and thus we can interchange the order of summation and have

$$
\begin{aligned}
\sum_{n=0}^{\infty} \lambda_{n} & =\sum_{k=1}^{\infty} \sum_{n=0}^{\infty}\left[r_{k+1}^{2 n+2}-2 s_{k}^{2 n+2}+r_{k}^{2 n+2}\right] \\
& =\sum_{k=1}^{\infty}\left[\frac{r_{k+1}^{2}}{1-r_{k+1}^{2}}-\frac{2 s_{k}^{2}}{1-s_{k}^{2}}+\frac{r_{k}^{2}}{1-r_{k}^{2}}\right]=\sum_{k=1}^{\infty} \frac{\left(2 s_{k}^{2}+r_{k+1} r_{k}+1\right)\left(r_{k+1}-r_{k}\right)^{2}}{2\left(1-r_{k+1}^{2}\right)\left(1-r_{k}^{2}\right)\left(1-s_{k}^{2}\right)}
\end{aligned}
$$

Writing $\delta_{n}=s_{k}-r_{k}=r_{k+1}-s_{k}$, it follows that

$$
\sum_{n=0}^{\infty} \lambda_{n} \approx \sum_{k=1}^{\infty} \frac{\delta_{k}^{2}}{\left(1-r_{k}\right)^{3}}=\sum_{k=1}^{\infty} \frac{\left(1 / k^{\alpha+1}\right)^{2}}{\left(1 / k^{\alpha}\right)^{3}}=\sum_{k=1}^{\infty} \frac{1}{k^{2-\alpha}}
$$

since $2 \delta_{k}=r_{k+1}-r_{k}=1 / k^{\alpha}-1 /(k+1)^{\alpha} \approx 1 / k^{\alpha+1}$, and the result follows.

Do these operators provide an example of a non-compact Toeplitz operator whose Berezin transform vanishes as we approach the boundary of the unit disk? The following recent result of Korenblum and Zhu [16] says that the answer is no.

THEOREM 6.4. Let $f$ be a bounded radial function on $\mathbb{D}$. Then

$$
T_{f} \text { is compact on } L_{a}^{2} \Leftrightarrow \tilde{f}(w) \rightarrow 0 \text { as }|w| \rightarrow 1^{-} \text {. }
$$

To prove the above theorem, let $f$ be a radial function on $\mathbb{D}$. Recall that the functions $e_{n}(z)=(n+1)^{1 / 2} z^{n}, n=0,1, \ldots$, form an orthonormal basis for $L_{a}^{2}$. It is easy to prove that $T_{f} e_{n}=\lambda_{n} e_{n}$, where

$$
\lambda_{n}=(n+1) \int_{\mathbb{D}} f(z)|z|^{2 n} d A(z) .
$$

Thus the operator $T_{f}$ is compact on $L_{a}^{2}$ if and only if $\lambda_{n} \rightarrow 0$ as $n \rightarrow \infty$. Note that equation (6.2) is a special case of (6.5). Using Proposition 1.2, the reproducing property of $K_{w}$, and (6.5) we obtain 


$$
\left\langle T_{f} K_{w}, K_{w}\right\rangle=T_{f} K_{w}(w)=\sum_{n=0}^{\infty} \overline{e_{n}(w)} T_{f} e_{n}(w)=\sum_{n=0}^{\infty} \lambda_{n}\left|e_{n}(w)\right|^{2}
$$

and thus we have the following formula for the Berezin transform:

$$
\widetilde{f}(w)=\widetilde{T_{f}}(w)=\left(1-|w|^{2}\right)^{2} \sum_{n=0}^{\infty}(n+1) \lambda_{n}|w|^{2 n}, \quad w \in \mathbb{D} .
$$

To prove Theorem 6.4 we need the following Tauberian theorem due to Hardy and Littlewood (Theorem 95 in [15]):

THEOREM 6.7. If $\left(b_{n}\right)$ is a bounded sequence of complex numbers such that

$$
(1-t) \sum_{n=0}^{\infty} b_{n} t^{n} \rightarrow 0 \quad \text { as } t \rightarrow 1^{-},
$$

then

$$
\frac{1}{n+1} \sum_{k=0}^{n} b_{k} \rightarrow 0 \quad \text { as } n \rightarrow \infty \text {. }
$$

Proof of Theorem 6.4. Suppose that $f$ is a radial function on $\mathbb{D}$ such that $\tilde{f}(w) \rightarrow 0$ as $|w| \rightarrow 1^{-}$. Then it follows from (6.6) that

$$
(1-t)^{2} \sum_{n=0}^{\infty}(n+1) \lambda_{n} t^{n} \rightarrow 0 \quad \text { as } t \rightarrow 1^{-} .
$$

Noting that

$$
(1-t)^{2} \sum_{n=0}^{\infty}(n+1) \lambda_{n} t^{n}=(1-t) \sum_{n=0}^{\infty}(n+1) \lambda_{n}\left(t^{n}-t^{n+1}\right)=(1-t) \sum_{n=0}^{\infty} b_{n} t^{n}
$$

where $b_{n}=(n+1) \lambda_{n}-n \lambda_{n-1}$ for $n \geq 1$, and $b_{0}=\lambda_{0}$, we have

$$
(1-t) \sum_{n=0}^{\infty} b_{n} t^{n} \rightarrow 0 \quad \text { as } t \rightarrow 1^{-},
$$

hence

$$
\frac{1}{n+1} \sum_{k=0}^{n} b_{k} \rightarrow 0 \quad \text { as } n \rightarrow \infty
$$

Using the fact that we have a telescoping sum we see that

$$
\frac{1}{n+1} \sum_{k=0}^{n} b_{k}=\lambda_{n}-\frac{\lambda_{0}}{n+1}
$$

Therefore $\lambda_{n} \rightarrow 0$ as $n \rightarrow \infty$, and it follows that $T_{f}$ is compact.

We can find another expression for the Berezin transform of $f$ by using (4.4) and the 
formula $|D(w, r)|=r^{2}\left(1-|w|^{2}\right)^{2} /\left(1-r^{2}|w|^{2}\right)^{2}$ (see, for example, Section 1.1 in [13]):

$$
\begin{aligned}
\tilde{f}(w) & =\sum_{k=1}^{\infty}\left(\int_{s_{k}<|z|<r_{k+1}} f\left(\varphi_{w}(z)\right) d A(z)+\int_{r_{k}<|z|<s_{k}} f\left(\varphi_{w}(z)\right) d A(z)\right) \\
& =\sum_{k=1}^{\infty}\left(\int_{D\left(w, r_{k+1}\right) \backslash D\left(w, s_{k}\right)} 1 d A(z)+\int_{D\left(w, s_{k}\right) \backslash D\left(w, r_{k}\right)}(-1) d A(z)\right) \\
& =\sum_{k=1}^{\infty}\left[\left|D\left(w, r_{k+1}\right) \backslash D\left(w, s_{k}\right)\right|-\left|D\left(w, s_{k}\right) \backslash D\left(w, r_{k}\right)\right|\right],
\end{aligned}
$$

thus

$$
\widetilde{f}(w)=\left(1-|w|^{2}\right)^{2} \sum_{k=1}^{\infty}\left[\frac{r_{k+1}^{2}}{\left(1-r_{k+1}^{2}|w|^{2}\right)^{2}}-\frac{2 s_{k}^{2}}{\left(1-s_{k}^{2}|w|^{2}\right)^{2}}+\frac{r_{k}^{2}}{\left(1-r_{k}^{2}|w|^{2}\right)^{2}}\right] .
$$

The above expression for the Berezin transform can be used to generalize Sarason's example. Recall that $2 \delta_{k}=r_{k+1}-r_{k}$.

THEOREM 6.9. Let $f$ be the function given by (6.1) with $s_{k}=\left(r_{k}+r_{k+1}\right) / 2$. If

$$
\sum_{k=1}^{\infty} \frac{\delta_{k}^{2}}{\left(1-r_{k}\right)^{2}}<\infty
$$

then $T_{f}$ is compact on $L_{a}^{2}$.

It follows from the above theorem that for every $\alpha>0$ the Toeplitz operator associated with $f$ is compact:

COROLlary 6.10. Let $f$ be the function given by (6.1) with $r_{k}=1-1 / k^{\alpha}$, where $\alpha>0$ and $s_{k}=\left(r_{k}+r_{k+1}\right) / 2$. Then $T_{f}$ is compact on $L_{a}^{2}$.

Pro of. Since $\delta_{k} \approx 1 / k^{\alpha+1}$, we have

$$
\frac{\delta_{k}^{2}}{\left(1-r_{k}\right)^{2}} \approx \frac{1 / k^{2 \alpha+2}}{1 / k^{2 \alpha}}=\frac{1}{k^{2}}
$$

Proof of Theorem 6.9. For fixed $w \in \mathbb{D}$ consider the function

$$
\psi(x)=x^{2} /\left(1-x^{2}|w|^{2}\right)^{2} .
$$

Then

$$
\psi^{\prime}(x)=2 x\left(1+x^{2}|w|^{2}\right) /\left(1-x^{2}|w|^{2}\right)^{3}
$$

and

$$
\psi^{\prime \prime}(x)=\left(2+16|w|^{2} x^{2}+6|w|^{4} x^{4}\right) /\left(1-x^{2}|w|^{2}\right)^{4} .
$$

Since $\psi^{\prime \prime}(x) \geq 0$, the graph of $\psi$ is convex, and thus $\psi\left(s_{k}\right) \leq\left(\psi\left(r_{k}\right)+\psi\left(r_{k+1}\right)\right) / 2$, hence $\psi\left(r_{k+1}\right)-2 \psi\left(s_{k}\right)+\psi\left(r_{k}\right) \geq 0$, proving that each of the terms in in the sum on the right-hand side of equation (6.8) is positive. Repeatedly using the mean value theorem we have

$$
\begin{aligned}
\psi\left(r_{k+1}\right)+\psi\left(r_{k}\right)-2 \psi\left(s_{k}\right) & \leq \delta_{k} 2 \delta_{k} \psi^{\prime \prime}\left(r_{k+1}\right) \leq \frac{48 \delta_{k}^{2}}{\left(1-r_{k+1}^{2}|w|^{2}\right)^{4}} \\
& \leq \frac{48 \delta_{k}^{2}}{\left(1-|w|^{2}\right)^{2}\left(1-r_{k+1}\right)^{2}} .
\end{aligned}
$$


For fixed $n \in \mathbb{N}$ it follows from (6.8) that

$$
\begin{aligned}
\tilde{f}(w) \leq & \left(1-|w|^{2}\right)^{2} \sum_{k=1}^{n}\left[\frac{r_{k+1}^{2}}{\left(1-r_{k+1}^{2}|w|^{2}\right)^{2}}-\frac{2 s_{k}^{2}}{\left(1-s_{k}^{2}|w|^{2}\right)^{2}}+\frac{r_{k}^{2}}{\left(1-r_{k}^{2}|w|^{2}\right)^{2}}\right] \\
& +48 \sum_{k=n+1}^{\infty} \frac{\delta_{k}^{2}}{\left(1-r_{k+1}\right)^{2}} .
\end{aligned}
$$

We conclude that

$$
\limsup _{|w| \rightarrow 1^{-}} \tilde{f}(w) \leq 48 \sum_{k=n+1}^{\infty} \frac{\delta_{k}^{2}}{\left(1-r_{k+1}\right)^{2}} .
$$

Taking the limit as $n \rightarrow \infty$ shows that $\tilde{f}(w) \rightarrow 0$ as $|w| \rightarrow 1^{-}$(recall that here $\tilde{f}(w) \geq 0$ for all $w \in \mathbb{D}$ ), and by Theorem 6.4 operator $T_{f}$ is compact on $L_{a}^{2}$.

In the above argument it is essential that $s_{k}$ is average of $r_{k}$ and $r_{k+1}$. If this is not the case, then the statement in the above theorem is false, as can be seen from the following example.

EXAMPLE 6.11. If $r_{k}=1-1 / k$ and $s_{k}=1-1 /\left(k+2^{-k}\right)=1-2^{k} /\left(1+k 2^{k}\right)$, then in [12] it is shown that the Hankel operator $H_{f}=H_{1+f}$ is compact on $L_{a}^{2}$ (actually Hilbert-Schmidt), and thus the operator $T_{f}$ cannot be compact on $L_{a}^{2}$ (for otherwise the multiplication operator $M_{f}$ would be compact, which would imply that $\widetilde{|f|^{2}}(w) \rightarrow 0$ as $|w| \rightarrow 1^{-}$, contradicting the fact that $|f|^{2}=1$ on $\left.\mathbb{D}\right)$.

Acknowledgments. This article is a revised and expanded version of my lectures in the semester "Linear Operators" held during the spring semester of 1994 at the Banach Center. I am very grateful to the organizers of the Semester for the invitation to visit and for providing local arrangements during my stay. I also acknowledge travel support from the University of Montana's faculty development program. I would like to thank Professor D. Sarason for supplying the details of his example [20] and Professor K. H. Zhu for sending me his recent paper with Professor B. Korenblum [16]. The final version of this article was written while visiting the Free University Amsterdam on sabbatical leave; I thank the University of Montana for awarding me a sabbatical and the Mathematics Department of the Free University for its hospitality and support.

Added in proof. S. Axler and D. Zheng have recently shown that for every $f \in L^{\infty}(\mathbb{D})$ the Toeplitz operator $T_{f}$ is compact on $L_{a}^{2}$ if and only if $\tilde{f}(w) \rightarrow 0$ as $|w| \rightarrow 1^{-}$.

\section{References}

[1] P. Ahern, M. Flores and W. Rudin, An invariant volume-mean-value property, J. Funct. Anal. 111 (1993), 380-397.

[2] J. Arazy, S. Fisher and J. Peetre, Hankel operators on weighted Bergman spaces, Amer. J. Math. 110 (1988), 989-1054.

[3] S. Axler, Bergman spaces and their operators, in: Surveys of Some Recent Results in Operator Theory, Vol. I, J. B. Conway and B. B. Morrell (eds.), Pitman Res. Notes, 1988, 1-50. 
[4] S. Axler, Berezin symbols and non-compact operators, unpublished manuscript, 1988.

[5] S. Axler and Ž. Čučković, Commuting Toeplitz operators with harmonic symbols, Integral Equations Operator Theory 14 (1991), 1-12.

[6] F. A. Berezin, Covariant and contravariant symbols of operators, Math. USSR-Izv. 6 (1972), 1117-1151.

[7] D. Békollé, C. A. Berger, L. A. Coburn and K. H. Zhu, BMO in the Bergman metric on bounded symmetric domains, J. Funct. Anal. 93 (1990), 310-350.

[8] C. A. Berger and L. A. Coburn, Toeplitz operators and quantum mechanics, ibid. 68 (1986), 273-299.

[9] - - - Toeplitz operators on the Segal-Bargmann space, Trans. Amer. Math. Soc. 301 (1987), 813-829.

[10] C. A. Berger, L. A. Coburn and K. H. Zhu, Function theory on Cartan domains and Berezin-Toeplitz symbol calculus, Amer. J. Math. 110 (1988), 921-953.

[11] A. Brown and P.R. Halmos, Algebraic properties of Toeplitz operators, J. Reine Angew. Math. 213 (1963), 89-102.

[12] J. A. Cima, K. Stroethoff and K. Yale, Bourgain algebras on the unit disk, Pacific J. Math. 160 (1993), 27-41.

[13] J. B. Garnett, Bounded Analytic Functions, Academic Press, New York, 1981.

[14] V. Guillemin, Toeplitz operators in n-dimensions, Integral Equations Operator Theory 7 (1984), 145-205.

[15] G.H. Hardy, Divergent Series, Clarendon Press, Oxford, 1949.

[16] B. Korenblum and K. H. Zhu, An application of Tauberian theorems to Toeplitz operators, J. Operator Theory 33 (1995), 353-361.

[17] J. Peetre, The Berezin transform and Ha-plitz operators, J. Operator Theory 24 (1990), $165-186$.

[18] P. Rosenthal, Berezin symbols and compactness of operators, unpublished manuscript, 1986.

[19] W. Rudin, Function Theory in the Unit Ball of $\mathbb{C}^{n}$, Springer, New York, 1980.

[20] D. Sarason, personal communication.

[21] J.H. Shapiro, The essential norm of a composition operator, Ann. of Math. 12 (1987), 375-404.

[22] K. Stroethoff, Compact Hankel operators on the Bergman space, Illinois J. Math. 34 (1990), 159-174.

[23] - Compact Hankel operators on the Bergman spaces of the unit ball and polydisk in $C^{n}$, J. Operator Theory 23 (1990), 153-170.

[24] - , Hankel and Toeplitz operators on the Fock space, Michigan Math. J. 39 (1992), 3-16.

[25] - Essentially commuting Toeplitz operators with harmonic symbols, Canad. Math. J. 45 (1993), 1080-1093.

[26] K. Stroethoff and D. Zheng, Toeplitz and Hankel operators on Bergman spaces, Trans. Amer. Math. Soc. 329 (1992), 773-794.

[27] K. H. Zhu, VMO, ESV, and Toeplitz operators on the Bergman space, ibid. 302 (1987), $617-646$

[28] - Operator Theory in Function Spaces, Dekker, New York, 1990. 